\title{
A IMPORTÂNCIA DO FARMACÊUTICO NO PRÉ E PÓS-OPERATÓRIO DE PACIENTES SUBMETIDOS À CIRURGIA BARIÁTRICA
}

\section{THE IMPORTANCE OF THE PHARMACIST IN THE PRE AND POSTOPERATIVE OF PATIENTS SUBMITTED TO BARIATRIC SURGERY}

\author{
DOMINGUES ${ }^{1}$, T. E.S; ASSUNÇÃO², D. P. S. F. \\ 1 - Acadêmica do Curso de Farmácia das Faculdades Ponta Grossa, Ponta Grossa-PR \\ 2 - Doutoranda pelo Programa de Pós-Graduação em Ciências Farmacêuticas Universidade Estadual de Ponta \\ Grossa, Ponta Grossa-PR.
}

Autor para correspondência: thaisespiritosanto@hotmail.com

\section{RESUMO:}

Os métodos cirúrgicos estão cada vez mais avançados, considerados eficazes para pacientes com obesidade mórbida. A primeira cirurgia bariátrica a ser realizada para 0 tratamento de obesidade foi realizada em 1954, por Kremen. Pacientes submetidos a cirurgia bariátrica são acompanhados por equipe multidisciplinar para auxiliar e motivar o tratamento bariátrico. O presente artigo teve como objetivo analisar a importância do papel do farmacêutico no tratamento pré e pós-operatório de pacientes, as formas farmacêuticas utilizadas, principais deficiências nutricionais, e relatos dos pacientes juntamente com suas expectativas e dificuldades. A presente pesquisa é classificada como exploratória, do tipo qualitativa e quantitativa, realizada através de questionário entregue a vinte pacientes de ambos os sexos, entre os meses de fevereiro a maio de 2017, em uma rede de farmácia de Telêmaco Borba-PR. Os dados obtidos foram analisados e agrupados no Microsoft Excel®. Todos os pacientes estavam com o IMC elevado, principal critério para intervenção cirúrgica. As principais técnicas em cirurgia bariátrica foram bypass gástrica em $Y$ de Roux e sleeve. A cirurgia bariátrica tem apresentado grande melhora na obesidade e principalmente na autoestima de pacientes, mas ainda existem dificuldades. O farmacêutico é o profissional habilitado e capacitado para esclarecer qualquer dúvida em relação aos medicamentos, seus efeitos colaterais e demais orientações sobre a cirurgia, devendo estar sempre presente no pré e pós-operatório de pacientes submetidos à cirurgia bariátrica.

Palavras-chave: Obesidade; cirurgia bariátrica; farmacêutico.

\begin{abstract}
:
Surgical methods are increasingly advanced, considered effective for patients with morbid obesity. The first bariatric surgery to be performed for the treatment of obesity was performed in 1954, by Kremen. Patients submitted bariatric surgery are accompanied by a multidisciplinary team to assist and motivate bariatric treatment. This article aimed to analyze the importance of the role of the pharmacist in the pre and postoperative treatment of patients, the pharmaceutical forms used, the main nutritional deficiencies, and patient reports together with their expectations and difficulties. The present research is classified as exploratory, of the qualitative and quantitative type, performed through a questionnaire delivered to twenty patients of both sexes, between the months of February to May 2017, in a pharmacy network of Telemaco Borba-PR. The obtained data were analyzed and grouped
\end{abstract}


in Microsoft Excel囚. All patients had high BMI, the main criterion for surgical intervention. The main techniques in bariatric surgery were Roux-en-Y gastric bypass and sleeve. Bariatric surgery has been shown great improvement in obesity and especially in the selfesteem of patients, but there are still difficulties. The pharmacist is qualified and qualified to clarify any doubts regarding medications, their side effects and other guidelines on the surgery, and should always be present in the pre and postoperative period of patients submitted bariatric surgery.

Keywords: Obesity; bariatric surgery; pharmacist.

\section{INTRODUÇÃO}

A obesidade é considerada um dos maiores problemas de saúde pública do mundo, de etiologia multifatorial e causada pelo acúmulo de gordura, sendo fator de risco para várias doenças (COSTA et al., 2009). Ainda, de acordo com a Pesquisa de Vigilância de Fatores de Risco e Proteção para Doenças Crônicas por Inquérito Telefônico (2017), mais da metade dos brasileiros estão com excesso de peso e 18,9\% estão obesos.

Existem vários métodos para classificação em obesidade, sendo utilizadas as medidas antropométricas, bioimpedância elétrica, pregas cutâneas e índice de massa corporal. As medidas antropométricas consistem nas dimensões físicas e composição global do corpo. A bioimpedância elétrica consiste em uma corrente elétrica de baixa intensidade conduzida através do corpo. Já as pregas cutâneas mede a espessura da gordura corporal localizada no tecido subcutâneo, devendo ser realizadas em várias regiões do corpo. (SANT'ANNA et al., 2009).

O Índice de Massa Corporal (IMC) é calculado pela divisão do peso (em quilos) pela altura (em metros) elevada ao quadrado. O índice de massa corpórea não está totalmente relacionado com a gordura corporal, porém tem sido amplamente utilizado e aceito para estudos epidemiológicos. (SOCIEDADE BRASILEIRA DE CIRURGIA BARIÁTRICA E METABÓLICA, 2017).

Estratégias para prevenir e tratar a obesidade englobam dietas, medicamentos e atividade física. Porém, vários pacientes não respondem a esses tratamentos tradicionais, necessitando assim de intervenção cirúrgica. (FANDIÑO et al., 2004).

Os métodos cirúrgicos estão cada vez mais avançados, considerados os mais eficazes para pacientes com obesidade mórbida. A cirurgia bariátrica é indicada para pacientes com IMC maior ou igual a $40 \mathrm{~kg} / \mathrm{m}^{2}$ ou maior que $35 \mathrm{~kg} / \mathrm{m}^{2} \mathrm{com}$ diabetes, apneia do sono, hipertensão arterial, dislipidemias e doenças coronarianas são grupos de pacientes que podem ser submetidos a cirurgia bariátrica. (SILVA et al., 2014).

A primeira cirurgia bariátrica a ser realizada para o tratamento de obesidade foi realizada em 1954, por Kremen. No Brasil, a evolução da cirurgia bariátrica ocorreu na 
década de 1970. O termo bariátrico deriva do grego baro, que significa peso, e iatros que significa os que praticam a medicina. (TAVARES et al., 2011).

As técnicas da cirurgia bariátrica são divididas em restritivas, mistas predominantes restritivas e mistas predominantes mal absortivas (disabsortiva). As restritivas têm a função de reduzir o tamanho do estômago sem interferir no intestino, diminuindo assim a quantidade de alimentos que o estômago pode armazenar e fazem parte desse grupo a banda ajustável e gastrectomia vertical, conhecida como sleeve (LIMA, 2012). A técnica mista predominante restritiva diminui principalmente o tamanho do estômago e reduz a superfície intestinal em contato com o alimento, o principal exemplo dessa técnica é a bypass gástrico em $Y$ de Roux (BORDALO et al., 2011). A técnica mista predominante disabsortiva consiste principalmente na diminuição da absorção dos nutrientes pelo intestino delgado, o qual é dividido em dois e redução do estômago, realizada pela técnica derivação biliopancreatica com gastrectomia horizontal e derivação biliopancreatica com duodenal switch. (ZEVE et al., 2012).

Segundo a Diretriz Brasileira de Obesidade (2009-2010), pacientes submetidos a cirurgia bariátrica são acompanhados por equipe multidisciplinar para auxiliar e motivar o tratamento bariátrico. A anamnese deve identificar fatores que contribuíram para o ganho de peso, quais as medicações e comorbidades, doenças patológicas, histórias psiquiátricas, nutricional e social. Os exames laboratoriais básicos e complementares são necessários para avaliações dos pacientes submetidos a cirurgia bariátrica.

Dentre a equipe multidisciplinar envolvida, encontra-se o farmacêutico com atuação importante no acompanhamento e educação aos pacientes, visando promover o uso racional de medicamentos, assegurando que os pacientes recebam o tratamento farmacológico apropriado e evitando que reações adversas interfiram nos resultados terapêuticos (CASTRO et al., 2006). Segundo Nunes et al. (2008), as intervenções farmacêuticas identificam problemas ocasionados por medicamentos e a prevenção dos problemas ocasionados pela grande maioria.

Considerando o aumento de procedimentos envolvendo a cirurgia bariátrica, este estudo tem como objetivo analisar a importância do papel do farmacêutico no tratamento pré e pós-operatório de pacientes, as formas farmacêuticas utilizadas, principais deficiências nutricionais e relatos dos pacientes juntamente com suas expectativas e dificuldades.

\section{MATERIAL E MÉTODOS}

Para realização desta pesquisa optou-se pelo método exploratório, do tipo 
qualitativa e quantitativa. $O$ instrumento da coleta de dados foi através de questionário com perguntas abertas e fechadas, entregues aos participantes após o preenchimento do Termo de Consentimento Livre e Esclarecido (TCLE) e Termo de Autorização de Uso de Imagem.

A pesquisa foi realizada com vinte indivíduos de ambos os sexos, na faixa etária de 20 a 60 anos, que foram submetidos à intervenção da cirurgia bariátrica independentemente do método, com tempo mínimo de cirurgia de um ano, que mostraram motivação para participar da pesquisa.

O local de pesquisa foi em uma rede de farmácia de Telêmaco Borba, devido ao grande fluxo de pacientes e a facilidade em realizar a pesquisa. $O$ período de coleta de dados foi realizado nos meses de fevereiro a maio de 2017.

Os dados obtidos após a aplicação dos questionários foram agrupados e analisados, utilizando aplicativo Microsoft Excel®. Conforme a resolução 466/12 do Conselho Nacional de Ética em Pesquisa, esta respeita todos os aspectos éticos tendo sido aprovado pelo Comitê de Ética em Pesquisa da FACPG, sob o número 1.941.373.

\section{RESULTADOS E DISCUSSÕES}

No presente estudo, dentre os participantes 14 são do gênero feminino (70\%) e 6 do gênero masculino (30\%), sendo dois de ensino fundamental, dez de ensino médio, três estudantes do ensino superior e cinco de ensino superior.

O método mais utilizado para classificar uma pessoa em obesa, é o cálculo do Índice de Massa Corpórea (IMC) e conforme mostra a Tabela 1, pode-se observar a classificação do IMC.

TABELA 1 - CLASSIFICAÇÃO DA OBESIDADE

\begin{tabular}{lc}
\hline \multicolumn{1}{c}{ Classificação } & IMC $\mathbf{~} \mathbf{g} / \mathbf{m}^{\mathbf{2}}$ \\
\hline Baixo peso (magra) & $<18,5$ \\
Normal & $18,5-24,9$ \\
Sobrepeso & $25-29,9$ \\
Obesidade leve (grau I) & $30-34,9$ \\
Obesidade moderada (grau II) & $35-39,9$ \\
Obesidade mórbida (grau III) & $>40$ \\
Superobesidade & $>50$ \\
\hline
\end{tabular}

Fonte: Sociedade Brasileira de Cirurgia Bariátrica e Metabólica, 2017. 
Através do cálculo, foram obtidos IMC de todos os pacientes antes da cirurgia e verificou-se que 7 pacientes (35\%) apresentavam IMC entre 35 a 39,9 kg/m², 10 pacientes (50\%) maior que $40 \mathrm{~kg} / \mathrm{m}^{2}$ e 3 pacientes (15\%) maior que $50 \mathrm{~kg} / \mathrm{m}^{2}$.

Novamente calculou-se o IMC, mas agora o atual dos pacientes, sendo que 4 pacientes (20\%) estão com IMC entre 18,5 a 24,9 kg/m², 11 pacientes (55\%) entre 25 a $29,9 \mathrm{~kg} / \mathrm{m}^{2}$, 3 pacientes (15\%) entre 30 a $34,9 \mathrm{~kg} / \mathrm{m}^{2}, 1$ paciente (5\%) entre 35 a $39,9 \mathrm{~kg} / \mathrm{m}^{2}$ e 1 paciente (5\%) acima de $40 \mathrm{~kg} / \mathrm{m}^{2}$. A Tabela 2 mostra a classificação do IMC antes da cirurgia e o atual dos pacientes, além do período de tempo da cirurgia.

TABELA 2 - CLASSIFICAÇÃO DO IMC ANTES DA CIRURGIA E O ATUAL E PERÍODO APÓS CIRURGIA

\begin{tabular}{ccccc}
\hline $\begin{array}{c}\text { IMC antes da } \\
\text { cirurgia } \mathbf{~ k g} / \mathbf{m}^{2}\end{array}$ & $\begin{array}{c}\text { Classificação IMC antes da } \\
\text { cirurgia }\end{array}$ & $\begin{array}{c}\text { IMC atual } \\
\mathbf{~ k g} / \mathbf{m}^{2}\end{array}$ & $\begin{array}{c}\text { Classificação IMC } \\
\text { atual }\end{array}$ & $\begin{array}{c}\text { Período após } \\
\text { cirurgia }\end{array}$ \\
\hline 54,93 & Superobesidade & 35,83 & $\begin{array}{c}\text { Obesidade moderada } \\
\text { (grau II) }\end{array}$ & 1 ano \\
44,39 & Obesidade mórbida (grau III) & 29,58 & Sobrepeso & 10 anos \\
38,01 & Obesidade moderada (grau II) & 23,31 & Normal & 7 anos \\
44,74 & Obesdade mórbida (grau III) & 24,65 & Normal & 3 anos \\
39,64 & Obesidade moderada (grau II) & 25,33 & Sobrepeso & 1 ano \\
42,29 & Obesidade mórbida (grau III) & 27,77 & Sobrepeso & 4 anos \\
38,86 & Obesidade moderada (grau II) & 26,64 & Sobrepeso & 7 anos \\
35,35 & Obesidade moderada (grau II) & 29,04 & Sobrepeso & 5 anos \\
39,86 & Obesidade moderada (grau II) & 25,48 & Sobrepeso & 5 anos \\
46,31 & Obesidade mórbida (grau III) & 27,38 & Sobrepeso & 2 anos \\
39,67 & Obesidade moderada (grau II) & 25,71 & Sobrepeso & 7 anos \\
41,52 & Obesidade mórbida (grau III) & 22,84 & Normal & 1 ano \\
38,93 & Obesidade moderada (grau II) & 24,24 & Normal & 4 anos \\
43,56 & Obesidade mórbida (grau III) & 30,3 & Obesidade leve (grau I) & 10 anos \\
51,19 & Superobesidade & 41,78 & Obesidade mórbida & (grau III) \\
53,99 & Sunorobesidade & 34,89 & Obesidade leve (grau I) & 6 anos \\
40,25 & Obesidade mórbida (grau III) & 26,4 & Sobrepeso & 6 anos \\
43,09 & Obesidade mórbida (grau III) & 34,15 & Obesidade leve (grau I) & 8 anos \\
43,09 & Obesidade mórbida (grau III) & 27,92 & Sobrepeso & 6 anos \\
43,75 & Obesidade mórbida (grau III) & 28,13 & Sobrepeso & 7 anos \\
\hline
\end{tabular}

Fonte: O autor, 2017.

Ao analisar o IMC de todos os pacientes antes da cirurgia, os índices de massa corpórea estavam entre obesidade moderada à superobesidade, predominando obesidade mórbida. Para submeter à cirurgia bariátrica os pacientes devem estar com IMC acima de 
$35 \mathrm{~kg} / \mathrm{m}^{2}$ ou acima de $40 \mathrm{~kg} / \mathrm{m}^{2}$ (SILVA et al., 2015). Sendo assim, todos os pacientes que foram submetidos à cirurgia bariátrica estavam dentro dos parâmetros necessários para a realização do procedimento cirúrgico.

A maioria dos pacientes perde entre 50 a $70 \%$ do excesso de peso após serem submetidos à cirurgia bariátrica, ocorrendo principalmente entre os primeiros meses e depois torna-se gradativo, estabilizando em aproximadamente 18 meses (QUÍRON, 2017). Segundo Bastos et al. (2013), o processo cirúrgico é considerado excelente se o IMC do paciente for menor que $30 \mathrm{~kg} / \mathrm{m}^{2}$, entre 30 a $35 \mathrm{~kg} / \mathrm{m}^{2}$ como bom resultado e maior que 35 $\mathrm{kg} / \mathrm{m}^{2}$ insucesso.

Para Barhouch (2010), a cirurgia bariátrica é considerada como sucesso se o paciente perder no mínimo $50 \%$ do excesso de peso ou mais até 5 anos após o procedimento cirúrgico. O excesso de peso e porcentagem da perda do excesso de peso é calculado através das Fórmulas 1 e 2.

Fórmula 1: Peso atual - peso ideal $\left(\mathrm{IMC} 25 \mathrm{~kg} / \mathrm{m}^{2}\right)=$ excesso de peso

$$
\text { Fórmula 2: } \frac{\text { Peso pré-operatório - peso atual }}{\text { Peso pré-operatório - peso ideal }} \times 100
$$

Após a cirurgia bariátrica, através do IMC atual de 15 pacientes $(75 \%)$ a cirurgia bariátrica foi considerada como excelente, de 3 pacientes (15\%) com bons resultados e 2 pacientes (10\%) ainda estão com o IMC acima de $35 \mathrm{~kg} / \mathrm{m}^{2}$, o que seria considerado como insucesso. Então foi realizado o cálculo do excesso de peso e da porcentagem da perda do excesso de peso dos dois pacientes com IMC acima de $35 \mathrm{~kg} / \mathrm{m}^{2}$, sendo que o paciente com IMC atual de $35,83 \mathrm{~kg} / \mathrm{m}^{2}$ com altura de $1,72 \mathrm{~cm}$ perdeu $63,82 \%$ do excesso de peso, tendo assim sucesso. Porém, o paciente com IMC atual de 41,78 kg/m² com altura de 1,63 perdeu 35,93\% do excesso de peso, não obtendo sucesso na cirurgia bariátrica.

Segundo Nicareta et al. (2015), o IMC é utilizado pela Organização Mundial da Saúde para classificar e comparar a obesidade. Aplicável a todos os indivíduos, de fácil interpretação no pós-operatório, da real perda de peso após a cirurgia e é recomendado para o seguimento pós-cirurgia.

Com relação aos tipos de técnicas escolhidas, 18 pacientes (90\%) foram submetidos à bypass gástrico em Y de Roux ou Fobi-Capella e 2 pacientes (10\%) à sleeve. As técnicas em cirurgia bariátrica são escolhidas pelo médico, baseado em informações 
sobre o mecanismo de funcionamento, resultados e riscos à cada paciente. A principal técnica do qual os pacientes foram submetidos, foi a bypass gástrica em $Y$ de Roux, também conhecido como Fobi-Capella. Técnica mais praticada devido a sua segurança sendo considerada como padrão-ouro, com resultados da perda de peso $75 \%$ do peso inicial e melhora do controle de comorbidades. (BORDALO et al., 2011)

Em relação ao tratamento utilizado antes de optar pela intervenção cirúrgica, 10 pacientes $(50 \%)$ realizaram atividade física, 13 pacientes $(65 \%)$ submeteram a algum tipo de dieta hipocalórica e 12 pacientes (55\%) utilizaram medicamentos, como a sibutramina. A sibutramina é um fármaco que atua na inibição da reabsorção, recaptação e degradação da noradrenalina, serotonina e dopamina, induzindo saciedade e aumentando o gasto energético termogênico. A sibutramina tem grande efetividade na perda de peso, tanto em pacientes com IMC superior a $35 \mathrm{~kg} / \mathrm{m}^{2}$ quanto em pacientes com IMC inferior a $35 \mathrm{~kg} / \mathrm{m}^{2}$. Porém, quando o fármaco é associado a atividade física e dietas hipocalóricas a redução de peso é potencializada. (MENEZES et al., 2010).

Os pacientes relataram as maiores dificuldades antes da cirurgia, sendo que 17 (85\%) pacientes relataram a perda de peso, 7 (35\%) pacientes a numeração e opção de roupas, $7(35 \%)$ pacientes a incidência de doenças crônicas e $6(30 \%)$ pacientes 0 emocional.

Identificou-se que 4 pacientes (20\%) faziam o uso de medicamentos para diabetes tipo 2, sendo que 2 pacientes (10\%) utilizavam metformina e 2 pacientes (10\%) utilizavam a insulina. Após a cirurgia bariátrica, a insulina foi substituída pela metformina em um dos usuários, o segundo apenas apresentou uma diminuição na dose de insulina. Nos dois pacientes que faziam uso da metformina, houve a suspensão do medicamento.

Segundo as Diretrizes da Sociedade Brasileira de Diabetes (2015-2016), 70\% a $90 \%$ dos pacientes submetidos à cirurgia bariátrica apresentavam redução do diabetes, com menores taxas em usuários de insulina. Já pacientes que utilizavam hipoglicemiantes orais, o diabetes é revertido após a cirurgia. Após a cirurgia bariátrica a melhora da resistência à insulina em pacientes com diabetes mellitus tipo 2 podem ocorrer pela alteração da expressão dos hormônios gastrointestinais, mudança da carga glicêmica ou diminuição do conteúdo lipídico. (KOHLI et al., 2015).

No pós-cirurgia 9 pacientes (45\%) desenvolveram patologias, sendo que 1 paciente (5\%) desenvolveu comportamentos compulsivos, 3 pacientes (15\%) depressão, 4 pacientes (20\%) refluxo gastroesofágico e 1 paciente (5\%) ulceração marginal. 
Segundo Machado et al. (2008), devido a redução do estômago, um componente restritivo é criado o qual diminui a capacidade de alimentos a serem ingeridos, dificultando à nova condição alimentar. A compulsão ocorre em pacientes ansiosos, depressivos e com o emocional prejudicado, sendo agravado após a operação.

A depressão também é relatada, definida como transtorno de humor, o qual o indivíduo perde o interesse ou prazer em realizar as atividades. A insatisfação corporal após a cirurgia pode gerar ou aumentar os sintomas de depressão. (ALMEIDA; ZANATTA; REZENDE, 2012).

O refluxo gastroesofágico é definido como a volta dos ácidos do estômago para o esôfago, devido ao esvaziamento gástrico acelerado após a cirurgia, secção ou diâmetro do anel de silicone colocado em cirurgia de bypass gástrica em Y de Roux. (FRAGA; MARTINS, 2012). Para o tratamento do refluxo gastroesofágico, o tratamento inicial é com os inibidores de bomba de prótons, o qual inibe a produção de ácidos pelas células parietais do estômago, reduzindo a agressão do esôfago. (HENRY, 2014).

A ulceração marginal ocorre devido ao surgimento de úlcera péptica próximo a anastomose gastrojejunal. De origem multifatorial, ao tamanho da bolsa, isquemia da mucosa, linha de grampeamento rompida, reação do corpo a estranhas substâncias exógenas. (PALERMO, 2015).

Quando realizada a cirurgia bariátrica, a capacidade gástrica é diminuída e consequentemente ocorre a redução da superfície intestinal. Além de interferir na absorção de nutrientes, aspectos farmacocinéticos e farmacodinâmicos de medicamentos também são interferidos pelas alterações fisiológicas do aparelho digestivo e mudanças metabólicas. (GORDON; KAIO; SALLET, 2011).

A escolha das formas farmacêuticas de medicamentos e suplementos após o procedimento cirúrgico tem grande importância, sendo que 14 pacientes (70\%) utilizaram injetáveis e comprimidos, 6 pacientes (30\%) utilizaram xarope, 4 pacientes (20\%) cápsulas e solução oral e 1 paciente (5\%) utilizou a via transdérmica.

A absorção de medicamentos diminui devido aos fármacos ácidos estarem em $\mathrm{pH}$ alto no trato digestório e a área de absorção ser menor. A melhor opção são fármacos com liberação imediata, como as injetáveis, pois são administradas diretamente na corrente sanguínea e também formas líquidas. Os comprimidos, cápsulas e drágeas devem ser pequenos, pois necessitam de ácido gástrico para liberação do fármaco e após a cirurgia o ácido gástrico é reduzido. (GORDON; KAIO; SALLET, 2011).

Os tipos de técnicas são os principais causadores de deficiências nutricionais. Pelo 
fato da técnica sleeve não ter a exclusão do duodeno, o sítio de absorção do ferro, cálcio e vitaminas do complexo $B$ não são interferidos. A técnica de bypass gástrica em $Y$ de Roux causa as maiores deficiências nutricionais, devido a remoção de grande parte do estômago, duodeno e jejuno, sendo os locais de absorção dos nutrientes. (ZEVE; NOVAIS; JÚNIOR, 2012).

Os pacientes submetidos à bypass gástrica em $\mathrm{Y}$ de Roux sofreram deficiências nutricionais, sendo que 15 pessoas (83,33\%) tiveram deficiência de ferro, 13 pacientes $(72,22 \%)$ de vitamina D, 7 pacientes $(38,9 \%)$ de vitamina B1 e 6 pacientes $(33,33)$ de cálcio. A vitamina B12, ácido fólico e proteínas tiveram deficiência menor aos pacientes, sendo 3 pacientes (16,68\%) com estas deficiências.

A deficiência do ferro em pacientes submetido a técnica de bypass gástrica em $Y$ de Roux ocorre devido a alterações na acidez gástrica, o que dificulta a conversão do ferro e a eliminação do seu sítio de absorção, o duodeno. (TRAINA, 2010).

O cálcio é absorvido no duodeno e jejuno, sendo facilitado pela vitamina $D$ em meio ácido e a vitamina $D$ é absorvida no jejuno e íleo, e ambos são comprometidos após a cirurgia bariátrica. Já as deficiências de vitamina B1 e proteínas podem ocorrer em pacientes submetidos a qualquer tipo de cirurgia bariátrica, pois estão relacionadas a vômitos prolongados, diarreia e intolerância alimentar que ocorrem após o procedimento cirúrgico. (BORDALO et al., 2011).

A vitamina B12 esta presente em quase todos os tecidos e esta envolvida na maturação das células vermelhas e sua deficiência pode ocasionar anemia macrocítica. A deficiência de vitamina B12 ocorre devido à falta do ácido gástrico para que seja liberada de uma fonte alimentar e a dificuldade de ser clivada no duodeno, para ligar-se no fator intrínseco e ser absorvida no íleo. Por causa da deficiência da vitamina B12, o ácido fólico não consegue ser convertido e consequentemente, resulta na sua deficiência. (ROCHA, 2012).

Como suplementação nutricional, dos 20 pacientes submetidos a cirurgia bariátrica, 6 pacientes $(30 \%)$ fazem o uso do Barivit $\circledast$ (polivitamínico e poliminerais), 4 pacientes $(20 \%)$ do Centrum ${ }^{\circledR}$ (polivitamínico e poliminerais), 3 pacientes $(15 \%)$ do Citoneurim $\AA$ comprimido (vitamina B12), 2 pacientes (10\%) utilizam a DePURA® (vitamina D) diariamente. A Cronobê® (vitamina B12) é utilizada por 2 pacientes (10\%) e 1 paciente $(5 \%)$ utiliza a Noripurum ${ }^{\circledR}$ intramuscular (ferro III), quando os exames detectam a deficiência destas vitaminas. Somente 2 pacientes (10\%) não fazem uso de complexo vitamínicos, ferro, complexo B ou ácido fólico, sendo estes submetidos a técnica sleeve, o que comprova que 
a técnica não altera a absorção das vitaminas.

Para controlar as deficiências nutricionais são realizados exames laboratoriais de hemograma, ureia e creatinina, glicemia em jejum, albumina, ferro sérico e ferritina.

O hemograma é um conjunto de avaliações das células do sangue, sendo composto por determinações básicas que incluem avaliações dos eritrócitos, dos leucócitos e das plaquetas (NAOUM; NAOUM, 2017). Segundo Santos et al. (2014), é um exame importante para verificar as causas da anemia, frequente em pacientes que foram submetidos a cirurgia bariátrica, devido à perda de sangue oculto ou pela modificação da absorção de nutrientes, como o ferro.

A avaliação do ferro sérico determina a concentração de ferro circulante no sangue (GROTTO, 2010). O ferro atua nas funções do metabolismo humano, transportando e armazenando oxigênio, e nas reações metabólicas, sendo estocado como ferritina e quando os valores de ferritina estão baixos indicam depleção do estoque de ferro devido à má absorção ou ingestão. (SANTOS et al., 2014).

Os exames de ureia e creatinina avaliam os rins e quando pacientes são submetidos à cirurgia bariátrica, podem apresentar elevados níveis de oxalúria o que levam a insuficiência renal. (MAZILI et al., 2017).

A ureia é produzida no fígado, circula no sangue, filtrada nos rins e excretada na urina. Em casos de não funcionamento dos rins, as impurezas irão se acumular, intoxicando o organismo pela ureia (CARVALHO, 2017). A creatinina é produto do metabolismo da creatinina muscular, o que varia de cada pessoa, e a eliminação pode ser por filtração glomerular ou secretada nos túbulos. Os valores aumentados de creatinina só aparecem quando pelo menos $50 \%$ da taxa de filtração glomerular (TFG) está diminuída, o que significa que precisa perder a função renal para os níveis se alterem. (CÂMARA, 2017).

A albumina é uma proteína plasmática, faz manutenção do volume plasmático circulante e em situações de insuficiência real, as suas concentrações de albumina ficam alteradas. (SANTOS et al., 2004).

O teste de glicemia em jejum consiste em medir a glicose no sangue após oito horas de jejum e a cirurgia bariátrica reduz a glicemia em jejum e melhora a resistência à insulina. (AYOUB; ALONSO; GUIMARÃES, 2011).

A frequência dos exames laboratoriais varia de três meses até um ano, sendo que 4 pacientes (20\%) realizam a cada três meses, 7 pacientes (35\%) a cada seis meses e 9 pacientes (45\%) pacientes uma vez ano.

Segundo as portarias Gabinete do Ministério (GM) / Ministério da Saúde (MS) 424 
e 425/2013, os exames devem ser realizados no $2^{\circ}$ mês, $6^{\circ}$ mês, $9^{\circ}$ mês, entre $12^{\circ}$ e $15^{\circ}$ meses e no 18ํㅡês após a cirurgia. Já Ramos e Mello (2015), recomendam que os exames ocorram anualmente.

Inúmeros são os relatos dos pacientes sobre os benefícios e as dificuldades após a cirurgia. Os benefícios em destaque foram a perda de peso e a melhora da autoestima. Já as dificuldades, cada paciente relata algo e todos os dados estão dispostos na Figura 1, a seguir.

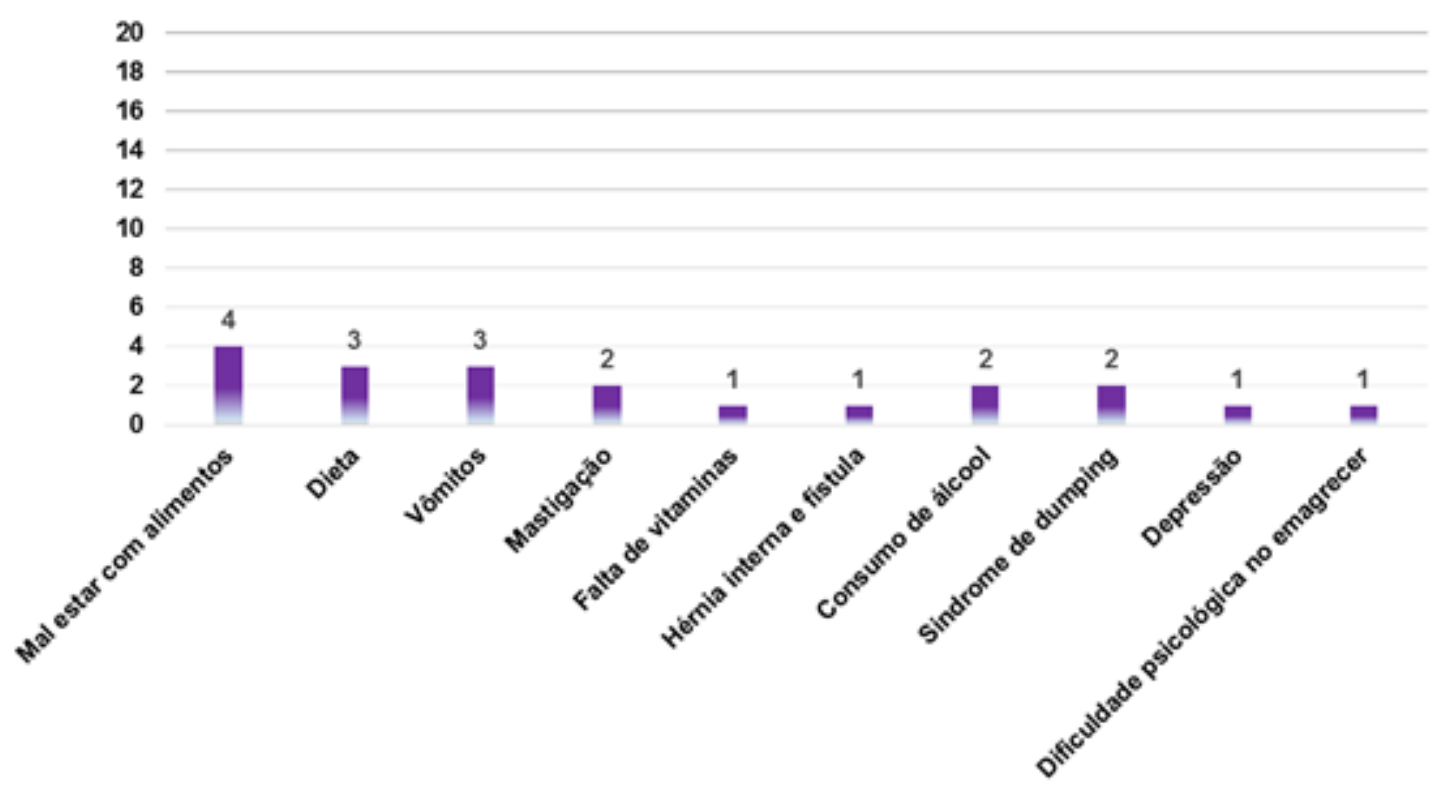

FIGURA 1 - DIFICULDADES APÓS A CIRURGIA BARIÁTRICA Fonte: O autor, 2017.

O desenvolvimento da fístula é uma grave complicação que pode ocorrer após a cirurgia bariátrica. Aumento da pressão intraluminal causado por estenose distal, tensão excessiva da linha da sutura e isquemia tecidual são fatores de risco para a causa da fístula (PERISSÉ et al., 2015). A hérnia interna é uma protusão do intestino dentro da cavidade abdominal (ACQUAFRESCA et al., 2015). O paciente que apresentou essas complicações, não conseguiu perder peso suficiente após a cirurgia.

A cirurgia bariátrica pode trazer consequências como a Síndrome de Dumping, devido ao esvaziamento rápido do estômago ao intestino delgado, levando a sintomas de palpitação, fadiga, calor, dor, náuseas e vômitos. Os sintomas estão relacionados a elevação dos níveis de insulina, seguidos de hipoglicemia (CHAVES, DESTEFANI, 2016).

O uso do álcool deve ter atenção maior em pacientes submetidos a cirurgia bariátrica, pois o paciente pode transferir sua compulsão por comida para o consumo 
excessivo do álcool (GREGORIO, 2016). Segundo Santos e Cruz (2016), consideram que o metabolismo do álcool é alterado facilitando sua dependência.

A depressão estava presente entre os pacientes submetidos a cirurgia bariátrica, sendo assim descrita anteriormente. A dificuldade psicológica no emagrecer muitas vezes afeta a perda de peso quando o alvo almejado parece distante, pois a imagem corporal se torna tão importante e o medo do reganho de peso tende a prevalecer (SILVA, 2015).

O acompanhamento clínico por uma equipe multidisciplinar é indispensável no pré e pós-operatório de pacientes submetidos a cirurgia bariátrica, sendo que 20 pacientes (100\%) tiveram a presença do médico cirurgião, 18 pacientes $(90 \%)$ da nutricionista, 15 pacientes (75\%) do psiquiatra, 9 pacientes (45\%) do fisioterapeuta e somente 4 pacientes (20\%) tiveram a presença do farmacêutico, conforme a Figura 2.

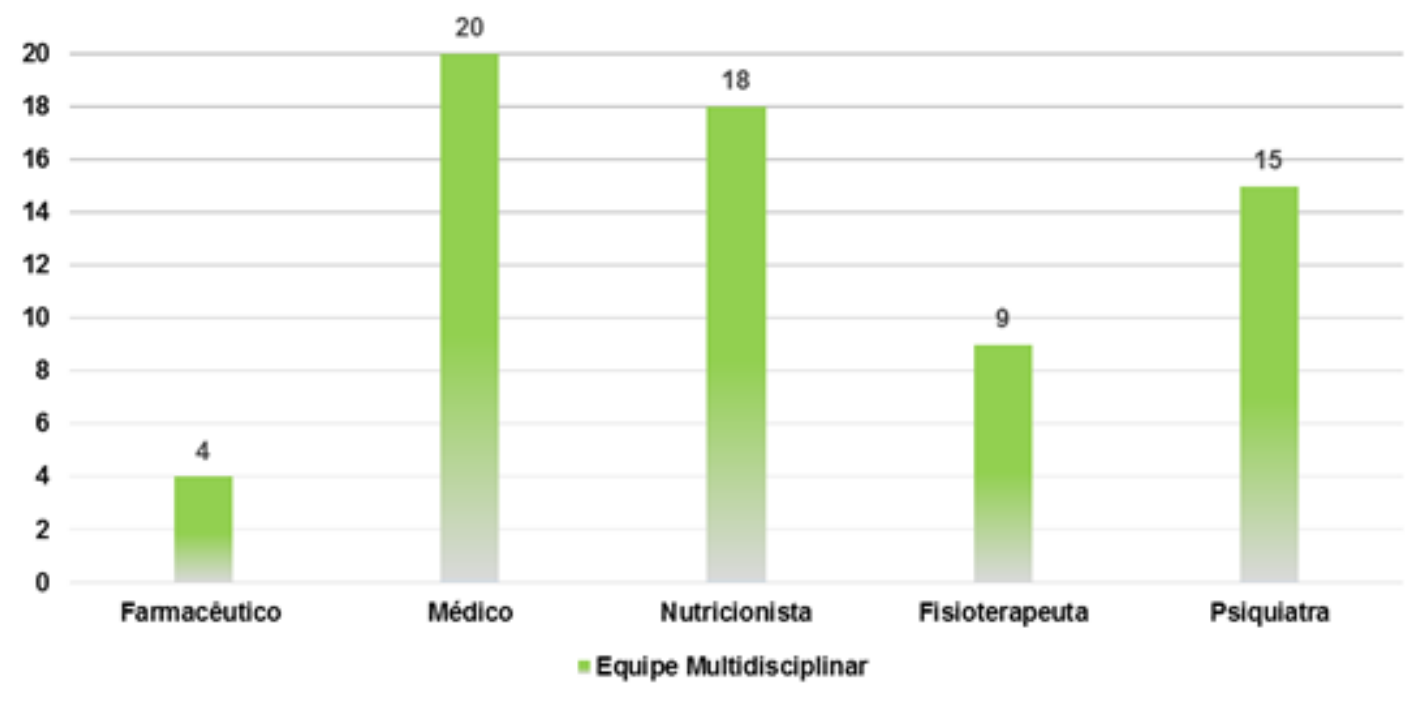

FIGURA 2 - EQUIPE MULTIDISCIPLINAR

Fonte: O autor, 2017.

Segundo Mulinari (2015), o farmacêutico desempenha um papel importante para otimizar o atendimento aos pacientes, avaliando os resultados de diversos medicamentos e evitando reações adversas.

O farmacêutico pode atuar em todas as etapas que envolvem medicamentos, desde a prescrição (colaborando com os médicos), dispensação, administração (informando à equipe de enfermagem a administração de medicamentos de forma segura) e monitoramento para garantir o uso seguro de medicamentos. (PILAU et al., 2014). 
O farmacêutico pode evitar possíveis erros de interpretação de receituário, identificando medicamentos que não foram prescritos ou inadequados para o procedimento cirúrgico. Erros relacionados as formas farmacêuticas também podem ser evitados, como a troca de comprimidos orais por liberação prolongada. (NUNES et al., 2008).

Pode orientar no pré-operatório a equipe multidisciplinar quanto a profilaxia de antimicrobianos, devido à alta incidência de infecção. Já no pós-operatório, o farmacêutico atua orientando os pacientes sobre a modificação da absorção de nutrientes, evitando deficiências nutricionais. (MULINARI, 2015).

O profissional farmacêutico pode também combater riscos relacionados a interações fármacos-nutrientes, ocasionando assim melhora da efetividade do tratamento farmacológico. É necessário orientações sobre as interações e ajuste dos horários das refeições e administração de medicamentos. (ANTUNES; LO PRETE, 2014).

O monitoramento da farmacoterapia pelo farmacêutico evita o uso de doses excessivas ou a não aderência ao tratamento, devido a utilização de vários medicamentos (KOVALESKI et al., 2016). Cabe ao farmacêutico o processo educativo sobre o risco da automedicação, interrupção do tratamento ou troca de medicamentos no pós-cirúrgico. (SPADA, 2017).

No entanto, o profissional farmacêutico ainda tem difícil aceitação por parte da equipe multidisciplinar, devido as diversas atuações o que geralmente ocorre a perda do foco clínico e atuação como profissional da saúde. (FREITAS et al., 2016).

\section{CONCLUSÃO}

Diante do exposto, conclui-se que a cirurgia bariátrica tem apresentado grande melhora na obesidade e principalmente na autoestima de pacientes, com satisfatória perda de peso por eles. Mas ainda apresentam dificuldades e complicações em alguns casos, como do paciente que mesmo após a cirurgia não obtiveram satisfatória perda de peso.

A cirurgia bariátrica modifica os sítios de absorção dos nutrientes e medicamentos, tendo assim que adaptar a melhor forma farmacêutica aos pacientes para evitar as deficiências nutricionais.

De maneira geral, analisando todos os pacientes desde o tratamento não cirúrgico, seguindo para a escolha da cirurgia bariátrica e no pré e pós-operatório, o farmacêutico é o profissional que pertence a todas essas áreas para auxiliar de forma direta e indireta. 
O farmacêutico é o profissional habilitado e capacitado para esclarecer qualquer dúvida em relação aos medicamentos, seus efeitos colaterais e demais orientações sobre a cirurgia, devendo estar sempre presente no pré e pós-operatório de pacientes submetidos a cirurgia bariátrica.

\section{REFERÊNCIAS}

ACQUAFrescA, P. A.; PAlERMO, M.; ROGUlA, T.; DUZA, G. E.; SERRA, E. Complicações cirúrgicas precoces após bypass gástrico: revisão da literatura. Arquivos Brasileiros de Cirurgia Digestiva. São Paulo, v. 28, n. 1, p.74-80, 2015.

ALMEIDA, S. S.; ZANATTA, D. P.; REZENDE, F. F. Imagem corporal, ansiedade e depressão em pacientes obesos submetidos à cirurgia bariátrica. Estudos de Psicologia. Campinas, v. 17, n. 1, p.153-160, 2012.

ANTUNES, A. O.; LO PRETE, A. C. O papel da atenção farmacêutica frente às interações fármaco-nutriente. Infarma. São Paulo, v. 26, n. 4, p. 208-214, 2014.

AYOUB, J. A. S.; ALONSO, P. A.; GUIMARÃES, L. M. V. Efeitos da cirurgia bariátrica sobre a síndrome metabólica. Arquivos Brasileiros de Cirurgia Digestiva. São Paulo, v. 24, n. 2, p.140-143, 2011.

BARHOUCH, A. S. Variação do percentual da perda do excesso de peso no pósoperatório tardio dos pacientes obesos mórbidos submetidos ao bypass gastrointestinal em y de Roux. 2010.50 p. Dissertação (Mestrado) - Curso de Medicina, Pontifícia Universidade Católica do Rio Grande do Sul, Porto Alegre, 2010.

BAstos, E. C. L.; BARBOSA, E. M. W. G.; SORIANO, G. M. S.; SANTOS, E. A.; VASCONCELOS, S. M. L. Fatores determinantes do reganho ponderal no pósoperatório de cirurgia bariátrica. Arquivos Brasileiros de Cirurgia Digestiva. São Paulo, v. 26, n. 1, p.26-32, 2013.

BORDALO, L. A.; TEIXEIRA, T. F. S.; BRESSAN, J.; MOURÃO, D. M. Cirurgia bariátrica: como e por que suplementar. Revista da Associação Médica Brasileira. Viçosa, v. 57, n.1, p.113-120, 2011. 
BRASIL. Ministério da Saúde. Portaria nำ424, de 19 de março de 2013.

BRASIL. Ministério da Saúde. Portaria n 425, de 19 de março de 2013.

CÂMARA, B. Brasil. Relação Ureia e Creatinina sérica. Disponível em: http://www.biomedicina padrao.com.br/2014/06/relacao-entre-ureia-e-creatinina-serica.html. Acesso em: 02 jun. 2017.

CARVALHO, E. M. Brasil. Ureia e Creatinina: exame da função dos rins. Disponível em: http://medifoco.com.br/ureia-e-creatinina-exame-da-funcao-dos-rins/. Acesso em: 02 jun. 2017.

CASTRO, M. S. D.; CHEMELLO, C.; PILGER, D.; JUNGES, F.; BOHNEN, L.; ZIMMERMAN, L. M.; PAULINO, M. A.; JACOBS, U.; FERREIRA, M. B. C.; FUCHS, F. D. Contribuição da atenção farmacêutica no tratamento de pacientes hipertensos. Revista Brasileira de Hipertensão, Rio de Janeiro, v. 13, n. 3, p.198-202, 2006.

CHAVES, Y. S.; DESTEFANI, A. C. Fisiopatologia, diagnóstico e tratamento da síndrome de dumping e sua relação com a cirurgia bariátrica. Arquivos Brasileiros de Cirurgia Digestiva. São Paulo, v. 29, n. 1, p.116-119, 2016.

COSTA, A. C. C.; IVO, M. L.; CANTERO, W. B.; TOGNINI, J. R. F. Obesidade em pacientes candidatos a cirurgia bariátrica. Acta Paulista de Enfermagem. Campo Grande, v. 22, n. 1, p.55-59, 2009.

DIRETRIZES DA SOCIEDADE BRASILEIRA DE DIABETES: 2015-2016. Sociedade Brasileira de Diabetes. São Paulo: AC Farmacêutica, 2015.

DIRETRIZES BRASILEIRAS DE OBESIDADE: 2009-2010. Associação Brasileira para o Estudo da Obesidade e da Síndrome Metabólica. 3.ed. Itapevi, SP: AC Farmacêutica, 2009.

FANDIÑO, J.; BENCHIMOL, A. K.; WALMIR, F. C.; APPOLINARIO, J. C. Cirurgia Bariátrica: aspectos clínico-cirúrgicos e psiquiátricos. Revista Psiquiatra. Rio Grande do Sul, v. 26, n. 1, p.47-51, 2004. 
FRAGA, P. L.; MARTINS, F. S. C. Doença do Refluxo Gastroesofágico: uma revisão de literatura. Cadernos UniFOA. Rio de Janeiro, v. 18, p.93-99, 2012.

FREITAS, G. R. M.; PINTO, R. S.; LUNA-LEITE, M. A.; CASTRO, M. S.; HEINECK, I. Principais dificuldades enfrentadas por farmacêuticos para exercerem suas atribuições clínicas no Brasil. Revista Brasileira de Farmácia Hospitalar e Serviços de Saúde. São Paulo, v. 7, n. 3, p.35-41, 2016.

GORDON, P. C.; KAIO, G. H.; SALLET, P. C. Aspectos do acompanhamento psiquiátrico de pacientes obesos sob tratamento bariátrico: revisão. Revista de Psiquiatria Clínica. São Paulo, v. 38, n. 4, p.148-154, 2011.

GREGORIO, V. D.; LUCCHESE, R.; VERA, I.; SILVA, G. C.; SILVA, A.; MORAES, R. C. C. O padrão de consumo de álcool é alterado após a cirurgia bariátrica? Uma revisão integrativa. Arquivos Brasileiros de Cirurgia Digestiva. São Paulo, v. 29, n. 1, p.111-115, 2016.

GROTTO, H. Z. W. Diagnóstico laboratorial da deficiência de ferro. Revista Brasileira de Hematologia e Hemoterapia. São Paulo, v. 32, n. 2, p.22-28, 2010.

HENRY, M. A. C. A. Diagnóstico e tratamento da doença do refluxo gastroesofágico. Arquivos Brasileiros de Cirurgia Digestiva. São Paulo, v. 27, n. 3, p.210-215, 2014.

KOHLI, R.; STEFATER, M.; INGE, T. H. Molecular insights from bariatric surgery. Reviews In Endocrine And Metabolic Disorders. EUA, p.1-11, 2015.

KOVALESKI, E. S.; SCHROEDER, H.; KRAUSE, M.; DANI, C.; BOCK, P. M. Perfil farmacoterapêutico de pacientes obesos no pós-operatório de cirurgia bariátrica. Jornal Vascular Brasileiro. São Paulo, v. 15, n. 3, p.182-188, 2016.

LIMA, L. F. Cirurgia bariátrica e melhora na qualidade de vida: revisão integrativa. 2012. 145 p. Monografia (Especialização) - Curso de Enfermagem, Universidade do Sul de Santa Catarina, Palhoça, 2012.

LOPES, E. M.; OLIVEIRA, E. A. R.; LIMA, L. H. O.; FORMIGA, L. M. F.; FREITAS, R. M. 
Interações fármaco-alimento/nutriente potenciais em pacientes pediátricos hospitalizados.

Revista de Ciências Farmacêuticas Básica e Aplicada. Araraquara, v. 34, n. 1, p.131-135, 2013.

MACHADO, C. E.; ZILBERSTEIN, B.; CECCONELLO, I. MONTEIRO, M. Compulsão alimentar antes e após a cirurgia bariátrica. Arquivos Brasileiros de Cirurgia Digestiva, São Paulo, v. 21, n. 4, p.185-191, 2008.

MAZILI, P. M. L.; NETO, E. V.; MANZANO, J. P.; ORTIZ, V. Brasil. Cirurgia bariátrica e litíase, fato ou ficção? Disponível em: http://www.moreirajr.com.br/revistas.asp?fase=r003\&id materia $=3717$. Acesso em: 02 jun. 2017.

MENEZES, C. A.; RIOS-SANTOS, F.; SANTOS, A. M. B.; SOUZA, M. E. A.; DI PIETRO, G. Efeito da sibutramina na redução do peso e no perfil metabólico em indivíduos obesos de uma população brasileira. Revista de Ciências Farmacêuticas Básica e Aplicada. Araraquara, v. 31, n. 3, p.159-164, 2010.

MULINARI, R. O papel do farmacêutico hospitalar no pré e pós-operatório de pacientes submetidos à cirurgia bariátrica. Revista Especialize On-line IPOG. Goiânia, v. 9, n. 10, p.1-17, 2015.

NAOUM, P. C; NAOUM, F. A. Brasil. Interpretação laboratorial do hemograma. Disponível em: http://www.ciencianews.com.br/arquivos/ACET/IMAGENS/Artigos_cientificos/ Interphemo.pdf. Acesso em: 02 jun. 2017.

NICARETA, J. R.; FREITAS, A. C. T.; NICARETA, S. M.; NICARETA, C.; CAMPOS, A. C. L.; NASSIF, P. A. N.; MARCHESINI, J. B. Análise crítica do método barros. Arquivos Brasileiros de Cirurgia Digestiva. São Paulo, v. 28, n. 1, p. 73-78, 2015.

NUNES, P. H. C.; PEREIRA, B. M. G.; NOMINATO, J. C. S.; ALBUQUERQUE, E. M.; SILVA, L. F. N.; CASTRO, I. R. S.; CASTILHO, S. R. Intervenção farmacêutica e prevenção de eventos adversos. Revista Brasileira de Ciências Farmacêuticas, São Paulo, v. 44, n. 8, p.691-699, 2008.

PALERMO, M. Complicações cirúrgicas tardias após bypass gástrico: revisão da literatura. 
Arquivos Brasileiros de Cirurgia Digestiva. São Paulo, v. 28, n. 2, p.139-143, 2015.

PÉRISSÉ, L. G. S.; PÉRISSÉ, P. C. M.; BERNARDO JÚNIOR, C. Tratamento endoscópico das fístulas após gastrectomia vertical e bypass gástrico em $Y$ de Roux. Revista do Colégio Brasileiro de Cirurgiões. Rio de Janeiro, v. 42, n. 3, p.159-164, 2015.

PILAU, R.; HEGELE, V.; HEINECK, I. Atuação do farmacêutico clínico em unidade de terapia intensiva adulto: uma revisão da literatura. Revista Brasileira de Farmácia Hospitalar e Serviços de Saúde. São Paulo, v. 5, n. 1, p.19-24, 2014.

QUÍRON. Brasil. Manual do Paciente. Disponível em: http://www.quironcirurgia.com.br/wpcontent/uploads/2015/05/manual_cir_bariatrica_quiron.pdf. Acesso em: 02 jun. 2017.

RAMOS, C. P.; MELLO, E. D. Manejo nutrológico no pós-operatório de cirurgia bariátrica. International Journal of Nutrology. S.I, v. 8, n. 2, p.39-49, 2015.

ROCHA, J. C. G. Deficiência de vitamina $B_{12}$ no pós-operatório de cirurgia bariátrica. International Journal of Nutrology. S.I, v. 5, n. 2, p. 82-89, 2012.

SANT'ANNA, M. S. L.; PRIORE, S. E.; FRANCESCHINI, S. C. Métodos de avaliação corporal em crianças. Revista Paulista de Pediatria. São Paulo, v. 27, n. 3, p. 315-321, 2009.

SANTOS, C. F.; AMADO, T.; SANDRI, Y. P.; FRIZZO, M. N. Alterações dos parâmetros laboratoriais em pacientes submetidos à cirurgia bariátrica. Saúde Integrada, S.I, p.9-30, 2014.

SANTOS, J. G.; CRUZ, M. S. Alcoolismo após cirurgia bariátrica: relato de caso. Jornal Brasileiro de Psiquiatria. São Paulo, v. 65, n. 4, p.340-343, 2016.

SANTOS, N. S. J.; DRAIBE, S. A.; KAMIMURA, M. A.; CUPPARI, L. Albumina sérica como marcador nutricional de pacientes em hemodiálise. Revista de Nutrição. Campinas, v. 17, n. 3, p.339-349, 2004.

SILVA, M. A. Emagrecimento induzido por cirurgia: ação do psicólogo após a cirurgia bariátrica. Boletim de Psicologia. Vitória da Conquista, v. 65, n. 143, p.243-246, 2015. 
SILVA, P. R. B.; SOUZA, M. R.; SILVA, E. M.; SILVA, S. A. Estado nutricional e qualidade de vida em pacientes submetidos à cirurgia bariátrica. Arquivos Brasileiros de Cirurgia Digestiva. São Paulo, v. 27, n. 1, p.35-38, 2014.

SOCIEDADE BRASILEIRA DE CIRURGIA BARIÁTRICA E METABÓLICA. Brasil. Press Kit. Obesidade sem marca. 2017.

SPADA, K. A função educativa do farmacêutico no sistema único de saúde. CBES. Porto Alegre, p. 1259-1270, 2017.

TAVARES, A.; VIVEIROS, F; CIDADE, C.; MACIEL, J. Cirurgia bariátrica do passado ao século XXI. Acta Médica Portuguesa. Vila Nova de Gaia, v. 24, n. 1, p.111-116, 2011.

TRAINA, F. Deficiência de ferro no paciente submetido à ressecção gástrica ou intestinal: prevalência, causas, repercussões clínicas, abordagem diagnóstica e prevenção. Revista Brasileira de Hematologia e Hemoterapia. São Paulo, v. 32, n. 2, p.78-83, 2010.

VIGITEL. Vigilância de fatores de risco e proteção para doenças crônicas por inquérito telefônico. Brasil. Hábitos dos brasileiros impactam no crescimento da obesidade e aumenta a prevalência de diabetes e hipertensão. Disponível em: http://portalarquivos. saude.gov.br/images/pdf/2017/abril/17/Vigitel.pdf. Acesso em: 02 jun. 2017.

ZEVE, J. L. M.; NOVAIS, P. O.; JÚNIOR, N. O. Técnicas em cirurgia bariátrica: uma revisão da literatura. Revista Ciência e Saúde. Porto Alegre, v. 5, n. 2, p.132-140, 201 